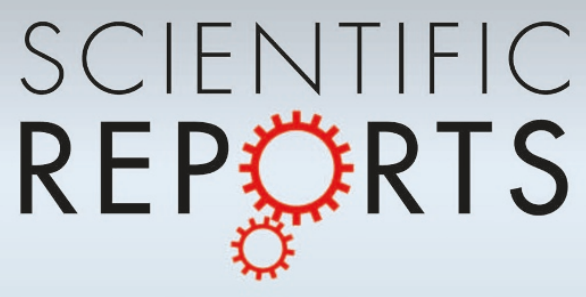

OPEN

SUBJECT AREAS:

APPLIED MICROBIOLOGY

EXPERIMENTAL MODELS OF

DISEASE

TRANSLATIONAL RESEARCH

Received

5 June 2014

Accepted

15 July 2014

Published

1 August 2014

Correspondence and requests for materials should be addressed to M.E. (mell@sund.ku.

$\mathrm{dk})$

\footnotetext{
* These authors contributed equally to this work.
}

\title{
Transfer of gut microbiota from lean and obese mice to antibiotic-treated mice
}

\author{
Merete Ellekilde * , Ellika Selfjord ' ${ }^{*}$, Christian S. Larsen', Maja Jakesevic', Ida Rune', Britt Tranberg', \\ Finn K. Vogensen ${ }^{2}$, Dennis S. Nielsen², Martin I. Bahl' ${ }^{3}$, Tine R. Licht ${ }^{3}$, Axel K. Hansen' \\ \& Camilla H. F. Hansen ${ }^{1}$
}

\begin{abstract}
'Department of Veterinary Disease Biology, Faculty of Health and Medical Sciences, University of Copenhagen, 1871 Frederiksberg C, Denmark, ${ }^{2}$ Department of Food Science, Faculty of Science, University of Copenhagen, 1958 Frederiksberg C, Denmark, ${ }^{3}$ National Food Institute, Technical University of Denmark, 2860 Søborg, Denmark.
\end{abstract}

Transferring gut microbiota from one individual to another may enable researchers to "humanize" the gut of animal models and transfer phenotypes between species. To date, most studies of gut microbiota transfer are performed in germ-free mice. In the studies presented, it was tested whether an antibiotic treatment approach could be used instead. C57BL/6 mice were treated with ampicillin prior to inoculation at weaning or eight weeks of age with gut microbiota from lean or obese donors. The gut microbiota and clinical parameters of the recipients was characterized one and six weeks after inoculation. The results demonstrate, that the donor gut microbiota was introduced, established, and changed the gut microbiota of the recipients. Six weeks after inoculation, the differences persisted, however alteration of the gut microbiota occurred with time within the groups. The clinical parameters of the donor phenotype were partly transmissible from obese to lean mice, in particularly $\beta$ cell hyperactivity in the obese recipients. Thus, a successful inoculation of gut microbiota was not age dependent in order for the microbes to colonize, and transferring different microbial compositions to conventional antibiotic-treated mice was possible at least for a time period during which the microbiota may permanently modulate important host functions.

n accelerating appreciation and exploration of our "forgotten organ" is increasingly advancing our understanding of how the gut microbiota can act as a regulator of human health. Before the infant is born, its gastrointestinal tract is sterile, but at birth and soon thereafter it acquires bacteria from the surrounding environment and the mother ${ }^{1}$. Once established, the microbiota remains relatively stable in the adult ${ }^{2}$ with a concentration of about $10^{11}$ colony forming units per gram feces ${ }^{3}$. However, environmental factors such as diets can cause shifts in the composition, known as dysbiosis, which in several studies have been associated with the onset of chronic inflammatory diseases such as diabetes ${ }^{4}$ and metabolic syndrome ${ }^{5}$. For example, changes in the gut microbiota have been associated with an obese phenotype, in particular increased Firmicutes:Bacteroidetes ratio in obese individuals compared to lean individuals ${ }^{5,6}$. Furthermore, in another study the Prevotella spp. (Bacteroidetes) to Eubacterium rectale ratio (Firmicutes) was positively correlated to plasma glucose levels ${ }^{7}$. It is reasonable to assume that the gut microbiota affects weight gain since it promotes increased uptake of monosaccharides and storage of triglyceride in adipocytes in the host and plays an essential role in processing of dietary polysaccharides ${ }^{8}$. This became apparent along with fecal transplantation experiments in which the transmissible dysbiosis from obese $l e p^{o b}$ and lean wild-type mice ${ }^{9}$ or from obese humans and their lean twins ${ }^{10}$ to adult germ-free (GF) mice demonstrated greater increase in body fat for mice colonized with an 'obese' gut microbiota. Moreover, GF mice, in contrast to conventionally raised mice, are resistant to increased body weight when fed a high fat diet ${ }^{11}$, demonstrating the importance of microbiota in obesity.

Especially gnotobiotic mice in which the contribution of a specific composition of gut microbes to disease development can be investigated have been useful to characterize potential immunological and metabolic pathways involved in disease pathogenesis. However, treating dysbiosis in co-housing experiments of rodents have also shown promising results; for example co-housing lean and obese mice resulted in a transfer of gut microbiota from the lean to the obese mice which prevented weight gain ${ }^{10}$. Also in humans, treating patients suffering from Clostridium difficile infection with stool transplant from healthy individuals was proven successful ${ }^{12}$.

Transferring microbiota was previously shown to be optimal at three weeks of age by inoculating GF mice which subsequently were housed in specific pathogen free (SPF) conditions ${ }^{13,14}$. In the present study, the feasibility of transferring gut microbiota from lean and obese mice to conventional mice was therefore investigated by 
reducing the existing gut microbiota with antibiotic treatment prior to inoculation. If possible, it would be a step further towards enabling researchers to manipulate gut microbiota and phenotype of animal models partly in place of more labour-demanding and expensive gnotobiotic mice. However, the timing of inoculation to conventional recipients may be $\mathrm{critical}^{14}$. Thus, two studies were performed: 1) A weaning study, in which $\mathrm{C} 57 \mathrm{BL} / 6$ dams were treated with ampicillin from one week prior to birth until weaning of the pups at three weeks of age, and pups were inoculated at weaning with a lean or obese mouse gut microbiota. 2) An adult study, in which C57BL/6 mice were inoculated at eight weeks of age, after three weeks of ampicillin treatment, with a lean or obese mouse gut microbiota. We thereby sought to investigate if gut microbiota transfer in antibiotic-treated mice was possible; if the gut microbiota of the recipients would resemble the donor microbiota they were given; if time of inoculation mattered and how stable the inoculum was in the recipients. The animals were furthermore monitored for parameters related to the metabolic phenotype of the donors.

\section{Results}

Gut microbiota differ between mice inoculated with lean and obese cecal content. Three weeks of ampicillin treatment prior to inoculation of cecal content from donors reduced the majority of bacterial groups in the gut. Ampicillin has previously been shown to be the drug that most consistently reduce gut bacterial density ${ }^{15}$ which was also evident by denaturing gradient electrophoresis (DGGE) profiles of $16 \mathrm{~S}$ rRNA gene PCR derived amplicons of fecal samples from the recipient mice, where only few bands emerged on the gel (Figure 1a). No difference in the gut microbiota before inoculation was evident between the groups, and the gut microbiota was equally reduced in both the adult treated mice and in the pups from antibiotic-treated mothers (not shown).

In the weaning study, the recipient mice clustered into two major groups one week after inoculation according to donor on the PCA analysis of fecal DGGE profiles, indicating a successful differentiation of either C57BL/6 or B6.-Lep ${ }^{o b}$ donor microbiota upon colonization (Figure 1b; $P<0.001$ for PC3 explaining $8.8 \%$ of the variance). There was still a significant difference in the DGGE fecal profiles between the B6.-Lep ${ }^{o b}$ and the lean $\mathrm{C} 57 \mathrm{BL} / 6$ recipient group two $(P<0.05$ for PC2 explaining $11.7 \%$ of the variance), three $(P<$ 0.05 for PC2 explaining $15.3 \%$ of the variance), four $(P<0.05$ for PC2 explaining $12.1 \%$ of the variance), and six (Figure 1c; $P<0.001$ for PC2 explaining $12.8 \%$ of the variance) weeks after inoculation (data not shown). Gender did not have any effect on the gut microbiota composition of the recipients.

The mice inoculated at eight weeks of age also clustered separately between the groups one week after inoculation (Figure 1d; $P<0.01$ for PC1 explaining $15.6 \%$ of the variance), though less related to the donors than was seen in the weaning study (the differences are in addition visualized in plots of PC values, Figure 1f-h). A group of mice receiving only PBS was included in the adult study and they were significantly different than the mice receiving $\mathrm{BALB} / \mathrm{c}$ (Figure $1 \mathrm{~d}+\mathrm{f} ; P<0.001$ for PC1) and B6.-Lep ${ }^{o b}$ donor microbiota (Figure $1 \mathrm{~d}+$ f; $P<0.01$ for PC1). They were most likely only slowly recolonized from the environment and therefore lacked several bands on the gel (Figure 11). No difference was observed in diversity between the obese and lean recipients in neither study at any time point. Six weeks after inoculation a significant difference in gut microbiota composition was still present between recipients of the obese B6.-Lep ${ }^{o b}$ and lean BALB/c donor microbiota (Figure $1 \mathrm{e}+\mathrm{j} ; P$ $<0.01$ for PC2 explaining $10 \%$ of the variation), and the mice that received PBS were still significantly different from the B6.-Lep ${ }^{o b}$ recipient mice (Figure $1 \mathrm{e}+\mathrm{j} ; P<0.01$ for $\mathrm{PC} 2$ and $P<0.01$ for PC3 explaining $9 \%$ of the variance) and BALB/c $(P=0.06$ for $\mathrm{PC} 3)$. There were no longer any clear difference in the diversity of the microbiota between the groups receiving donor microbiota and PBS (Figure 1m).

Taxonomic differences between mice inoculated with B6.-Lep ${ }^{o b}$ or C57BL/6 microbiota does not persist. To investigate the effect of the inoculations further, a comparison of different taxa in the microbiota of donors and recipients was performed in the weaning study. The abundances of bacterial taxa in the microbiota profiles of the two donors revealed a lower abundance of Bacteroidetes (Figure $2 \mathrm{f}$ ) and Bacteroides (Figure $2 \mathrm{~g}$ ) in the B6.-Lep ${ }^{o b}$ donor compared to the lean $\mathrm{C} 57 \mathrm{BL} / 6$ donor. One week after inoculation, the B6.-Lep ${ }^{o b}$ recipients also had a significantly lower abundance of Bacteroidetes but not the Bacteroides, and a significantly higher abundance of $B$. fragilis (Figure 2h) and Enterobacteriaceae (Figure 2k) was evident. Six weeks after inoculation these differences were no longer present. In mice inoculated with C57BL/6 donor microbiota, a tendency towards a reduction in the taxa Bacteroides and Parabacteroides diastonis from one to six weeks after inoculation was evident, thus the microbiota changed over time after inoculation (Figure $2 \mathrm{~m}, P=$ 0.083 and $P=0.095$, respectively) as also observed in the PCA plots of DGGE profiles. In recipients of the B6.-Lep $p^{o b}$ donor microbiota, significant increases in the abundance of Firmicutes and Lactobacillus spp., and a decrease in B. fragilis were observed from one to six weeks post inoculation (Figure 2n; $P<0.05$ ). Furthermore, a tendency towards an increase in Bacteroidetes $(P$ $=0.095)$ and a decrease in Enterobactericeae $(P=0.089)$ was evident, which likely explain the lack of difference between the B6.-Lep ${ }^{o b}$ and C57BL/6 recipients six weeks post inoculation.

Recipients of B6.-Lep ${ }^{o b}$ microbiota have increased $\beta$ cell response to glucose despite only minor increase in weight gain and fat deposition. Males and females were significantly different in body weight (Figure 3a-b; $P<0.001$ and Figure $4 \mathrm{a}-\mathrm{b} ; P<0.01$ )) and total food consumption (Figure $3 \mathrm{c} ; P<0.05$, and Figure $4 \mathrm{c} ; P<0.0001$ ). In addition, both male and female recipient mice of the obese microbiota had a higher weight gain than the lean recipients inoculated as adults, whereas this was not evident in the mice inoculated at weaning. Food consumption was furthermore elevated in male B6.-Lep ${ }^{o b}$ recipient mice inoculated at weaning compared to lean recipients whereas this was not significantly different in the mice inoculated as adults $(n=2)$. Food consumption in one of the cages with four female BALB/c recipient mice was lower than the B6.-Lep ${ }^{o b}$ recipient mice, but not in the other cage, and thus not significant on cage level. There was also a gender difference in total weight of body fat pads $(P<0.05$, Figure $3 \mathrm{c})$ and a strong tendency for larger body fat pads was found in the male B6.-Lep ${ }^{o b}$ recipients compared to lean microbiota recipients inoculated at weaning $(P=0.057)$.

No effect of inoculation on glycated hemoglobin (HbA1c\%), a measure of the stable blood glucose concentration over time, was observed in neither the young nor the adult inoculated mice (Figure $3 \mathrm{e}$ and Figure $4 \mathrm{~d}$ respectively). There seemed to be no difference in insulin resistance between the groups in the glucose tolerance tests as both groups similarly cleared the high glucose level, however the peak of blood glucose was significantly or borderline higher in the lean microbiota recipient mice compared to B6.-Lep ${ }^{o b}$ recipient mice 4-6 weeks after inoculation at both weaning and eight weeks of age (Figure $3 \mathrm{f}-\mathrm{h}$ and Figure $4 \mathrm{e}$ ). AUC was furthermore significantly lower $(P<0.05)$ in the B6.-Le $p^{o b}$ recipient mice four weeks after inoculation at weaning compared to the lean recipient group. The increase in serum insulin level after inoculation at weaning was significantly higher in $\mathrm{B} 6 .-L e p^{o b}$ recipient mice compared to recipient mice of lean microbiota $(P<0.05$; Figure $3 i)$.

\section{Discussion}

There are many indications of a causative role of the gut microbiota in the development of obesity, glucose homeostasis, and insulin res- 


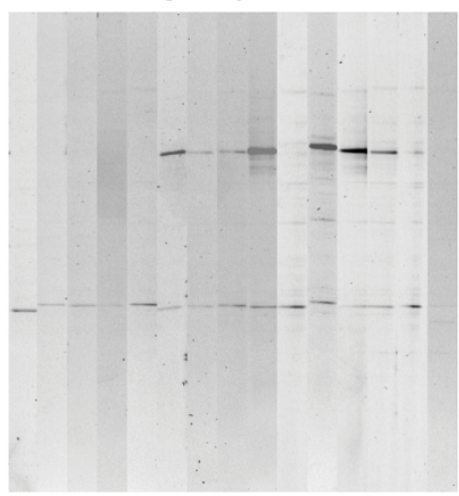

d b

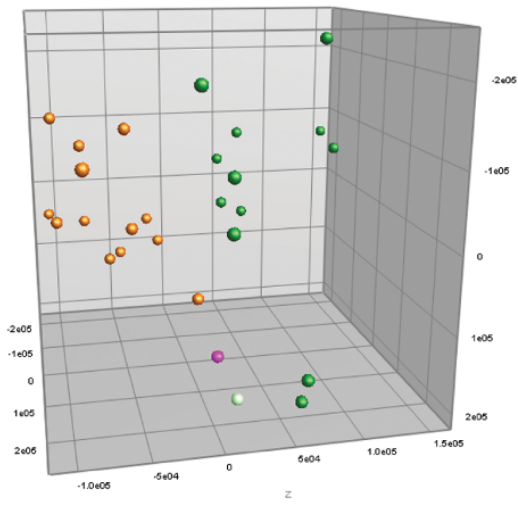

e

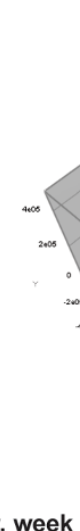

Adult study, week 6

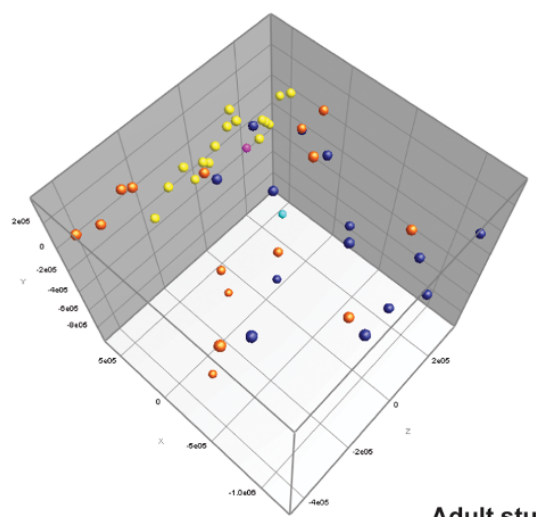

f

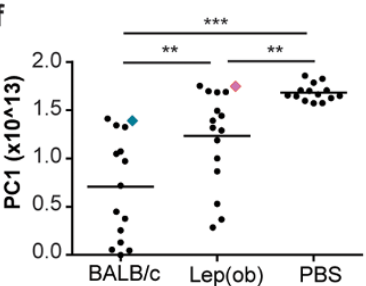

g

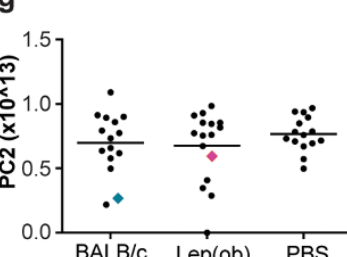

h

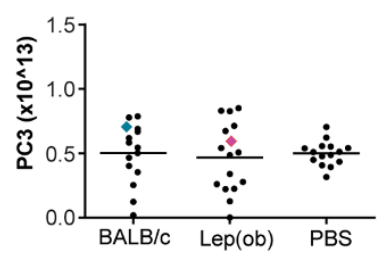

C

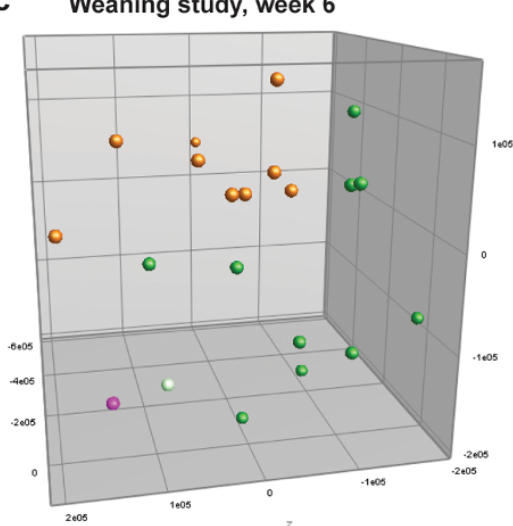

I
Adult study, week 1

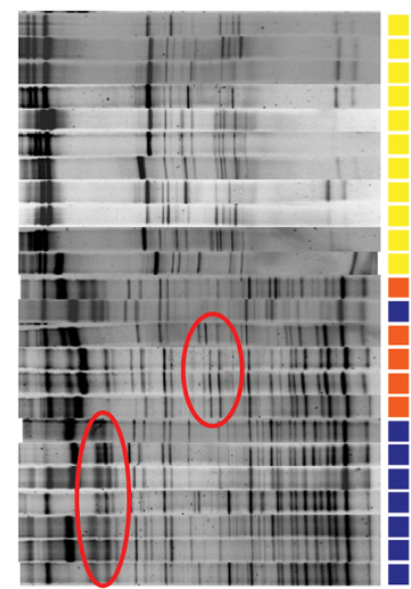

m

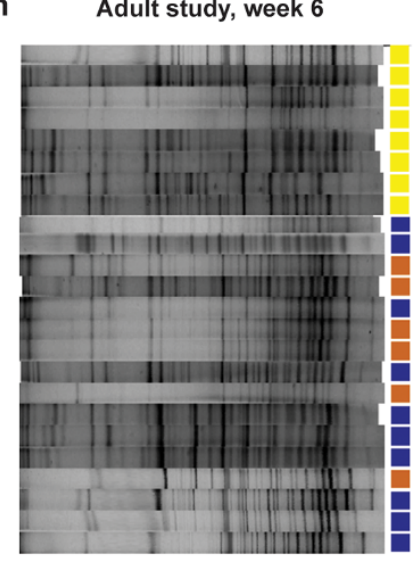

Figure 1 Gut microbiota analysis by denaturing gradient gel electrophoresis (DGGE). (a) Feces samples were collected from ampicillin-treated B6 recipient mice just before inoculation. The picture shows a segment of a gel illustrating DGGE profiles of three weeks old ampicillin-treated mice before they were randomized to receive microbiota inoculation from donor mice. Each lane represents the gut microbiota profile of one mouse. (b) Principal Component Analysis (PCA) plot of DGGE profiles from feces samples collected from recipient mice one week after inoculation at three weeks of age with either C57BL/6 (green balls $n=12$; donor: light green ball $n=1$ ) or B6.- Lep ${ }^{o b}$ (orange balls $n=13$; donor: pink ball $n=1$ ) microbiota. (c) PCA plot of DGGE profiles from feces samples collected from recipient mice six weeks after inoculation at three weeks of age with either C57BL/6 (green balls $n=12$; donor: light green ball $n=1$ ) or B6.-Lep $p^{o b}$ orange balls $n=13$; donor: pink ball $n=1$ ) microbiota. (d) PCA plot of DGGE profiles from feces samples collected from recipient mice one week after inoculation at eight weeks of age with either BALB/c (dark blue balls $n=14$; donor: light blue ball $n=1$ ), B6.-Lep ${ }^{o b}$ (orange balls $n=15$; donor: pink ball $n=1$ ) microbiota, or PBS (yellow balls $n=14$ ). (e) PCA plot of DGGE profiles from feces samples collected from recipient mice six weeks after inoculation at eight weeks of age with either BALB/c (dark blue balls $n=14$; donor: light blue ball $n=1$ ), B6.-Lep ${ }^{o b}$ (orange balls $n=13$; donor: pink ball $n=1$ ) microbiota, or PBS (yellow balls $n=15)$ ). (f-h) Scatterplots of PC1 (x-component), PC2 (y-component), and PC3 (z-component) respectively from DGGE profiles of feces samples shown in D. (i-k) Scatterplots of PC1 (x-component), PC2 (y-component), and PC3 (z-component) respectively from DGGE profiles of feces samples shown in E. (1) The picture shows a segment of a gel illustrating DGGE profiles of mice one week after inoculation with BALB/c microbiota (blue), B6.-Lep ${ }^{o b}$ microbiota (orange), or PBS (yellow). The red circles mark examples of differences in appearance of bands between the two inoculated groups. $(\mathrm{m})$ The picture shows a segment of a gel illustrating DGGE profiles of mice six weeks after inoculation with BALB/c microbiota (blue), B6.-Lep ${ }^{o b}$ microbiota (orange), or PBS (yellow). 
a

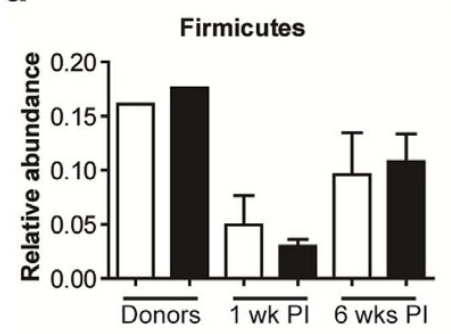

d

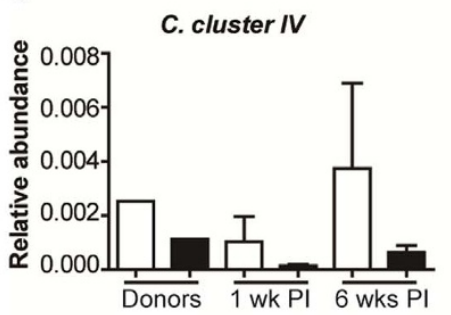

g
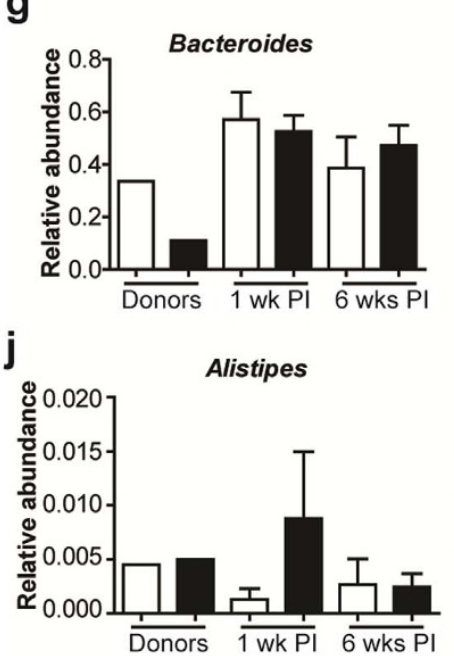

b

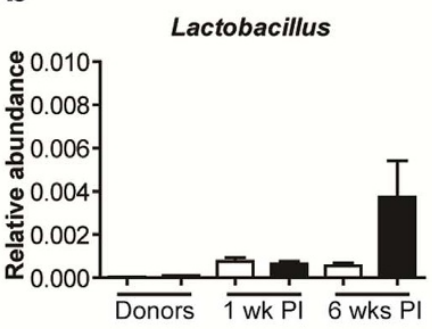

e

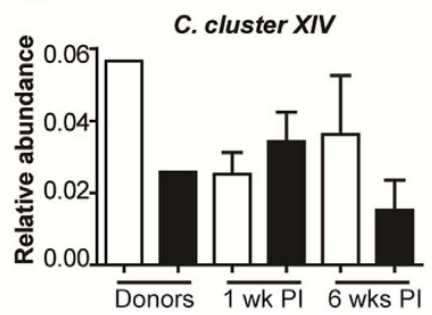

h

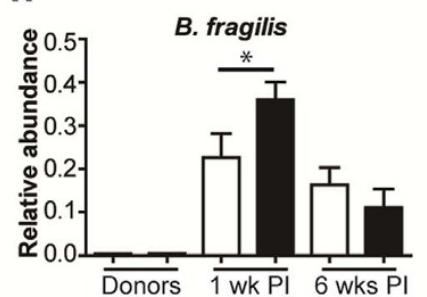

k

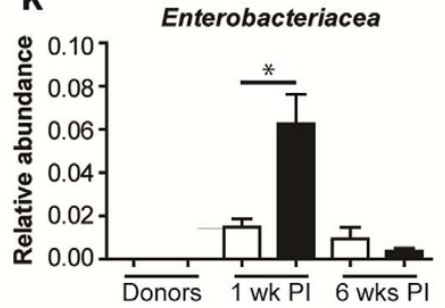

C

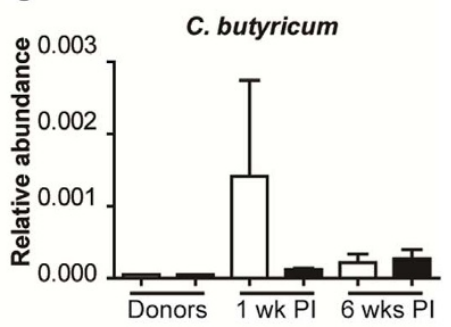

f

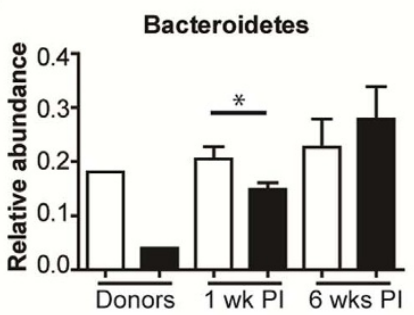

i

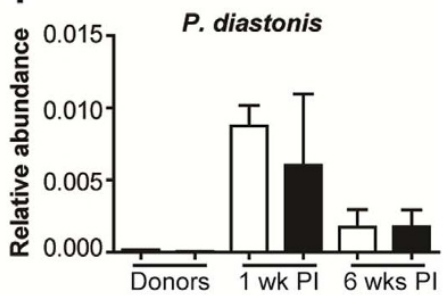

I

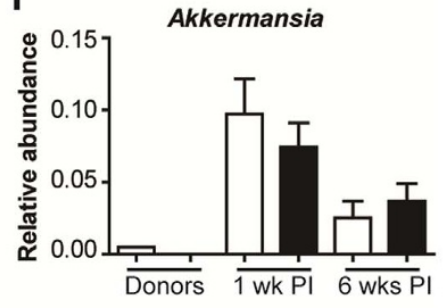

m

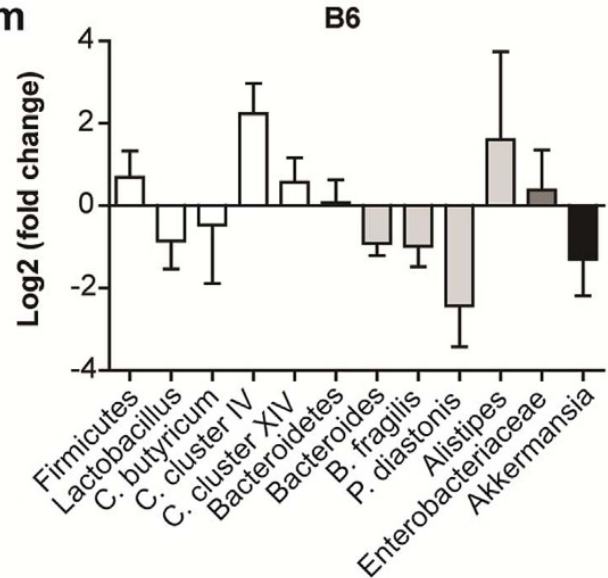

n

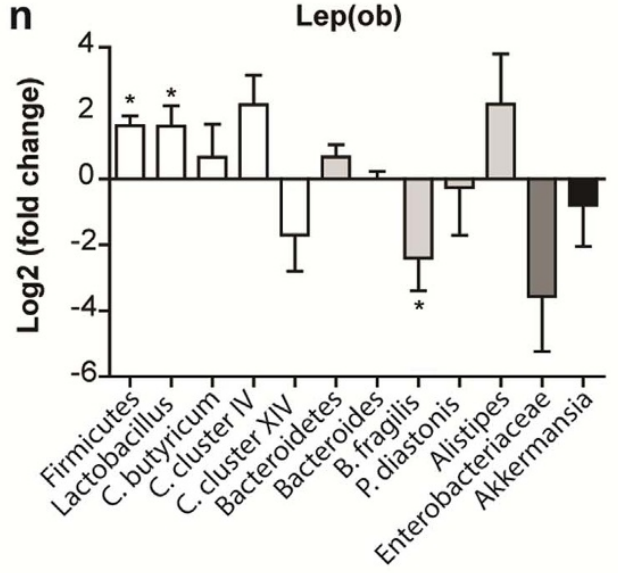

Figure 2 Quantitative gut microbiota comparison of taxa derived from analysis with GUt Low-Density Array (GULDA). (a-1) Relative abundance of selected 16S rRNA gene targets with given microbial primers. Cecum samples from C57BL/6 $(n=1)$ and B6.-Lep ${ }^{o b}(n=1)$ donor mice and fecal samples from recipient mice one week and six weeks after inoculation at three weeks of age with either C57BL/6 (white bars, $n=10$ ) or B6.-Lep $p^{o b}$ microbiota (black bars, $n=13$ ) are shown. (m) Ratio (fold-change) of selected 16S rRNA gene targets in feces from recipient mice at one week to six weeks after inoculation at three weeks of age with C57BL/6 microbiota $(n=4)$. Different shading of columns represent the four phyla Firmicutes (white),

Bacteroidetes (light grey), Proteobacteria (grey), Verrucomicrobia (black). $\mathrm{P}<0.1$ for a hypothetical value different from 0 (no change) is indicated. (n) Ratio (fold-change) of selected $16 \mathrm{~S}$ rRNA gene targets in feces from recipient mice at one week to six weeks after inoculation at three weeks of age with B6.-Le $p^{o b}$ microbiota $(n=5)$. All samples analyzed were quantified in duplicate. The relative abundance values are derived by normalizing with a universal bacterial primer-target. The bar charts show means \pm SEM. * represents $P<0.05$. 


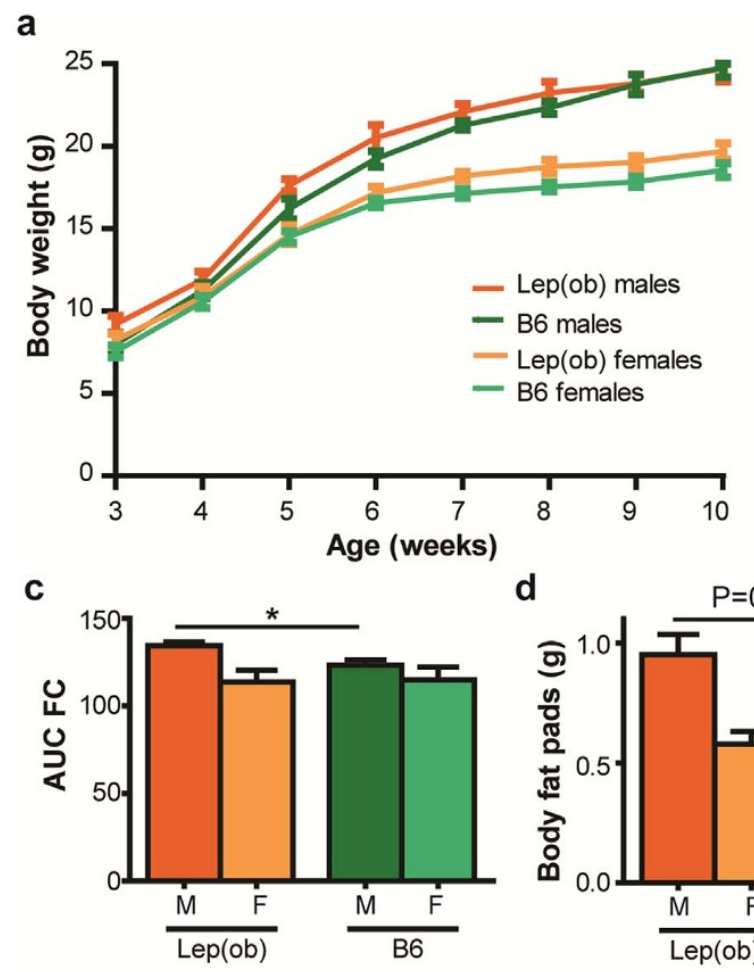

b
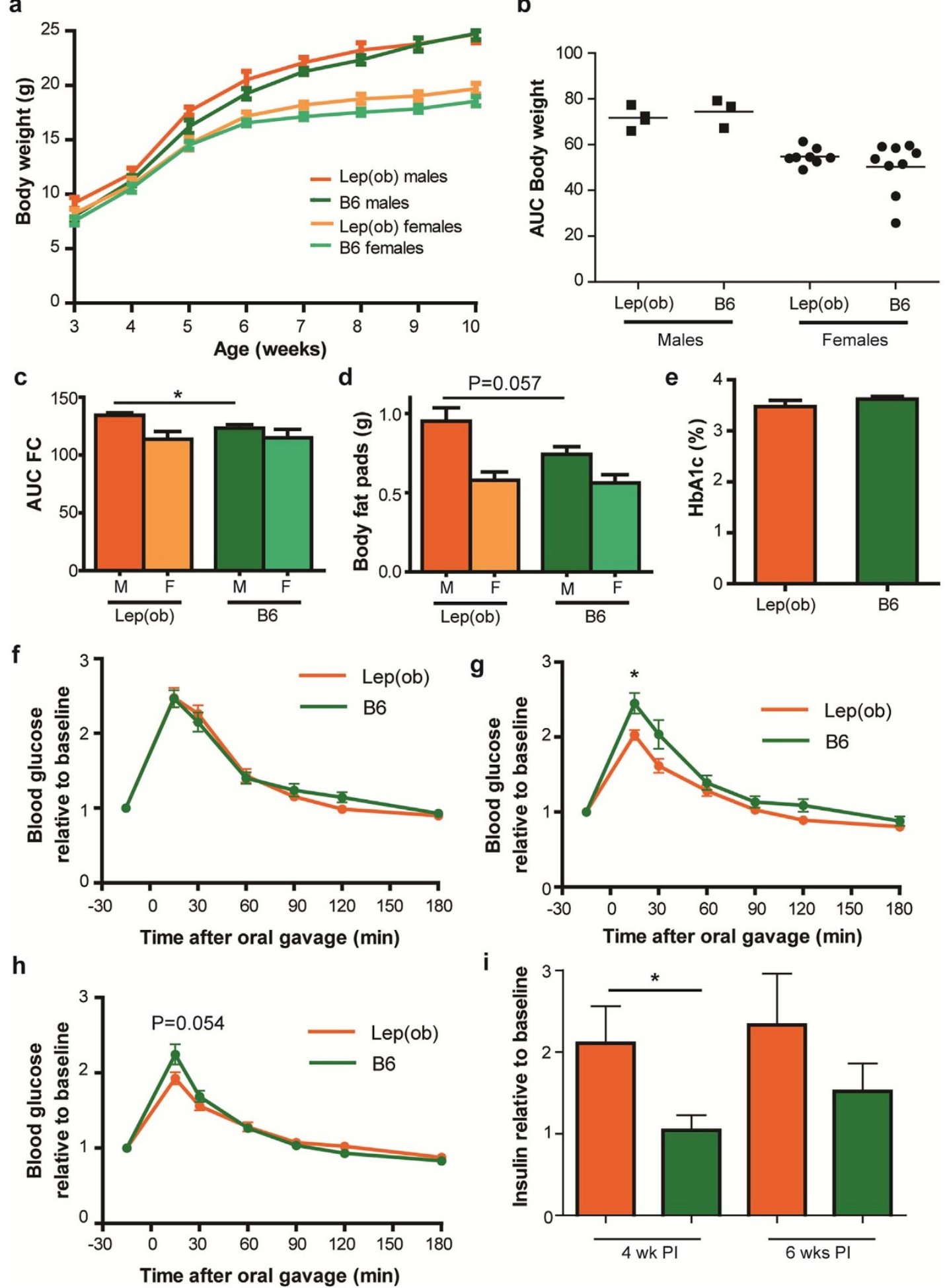

Figure 3 Microbiota transfer at weaning changes metabolic parameters in antibiotic-treated recipient mice. (a) Weekly body weight (gram) of recipient mice after inoculation with either C57BL/6 (males $n=3$; females $n=7$ ) or B6.-Lep ${ }^{o b}$ microbiota (males $n=4$; females $n=8$ ) at three weeks of age. (b) Area under the curve (AUC) calculated from weight data in A. (c) AUC of weekly food consumption (gram) per mouse in average per cage in six weeks from inoculation in recipient mice inoculated with either C57BL/6 (males $n=2$; females $n=4$ ) or B6.-Lep ${ }^{o b}$ microbiota (males $n=4$; females $n=3$ ) at three weeks of age. (d) Weight (gram) of body fat pads (the sum of inguinal fat pads, retroperitoneal pad pads, epididymal fat pads or uterine fat pads in males and females respectively) in recipient mice six weeks after inoculation with either C57BL/6 (males $n=3$; females $n=9$ ) or B6.-Lep $p^{o b}$ microbiota (males $n=4$; females $n=8$ ) at three weeks of age. (e) HbAlc (glycated haemoglobin, \%) concentration in the blood of recipient mice six weeks after inoculation with either C57BL/6 ( $n=9$, green bars) or B6.- Lep $p^{o b}(n=11$, orange bars) microbiota at three weeks of age. (f-h) Oral glucose tolerance test (OGTT) one, four, and six weeks (respectively) after inoculation with either C57BL/6 $(n=12)$ or B6.-Lep ${ }^{o b}(n=12)$ microbiota at three weeks of age. Tail blood glucose relative to baseline value $(-15 \mathrm{~min}$ ) is shown at given time points after oral gavage of glucose. * represents $P<0.05$ difference in peak values. (i) Fasting blood insulin levels relative to insulin levels at inoculation at three weeks of age. Insulin levels are shown four and six weeks post inoculation (PI). * represents $P<0.05$. Mean \pm SEM. 
a

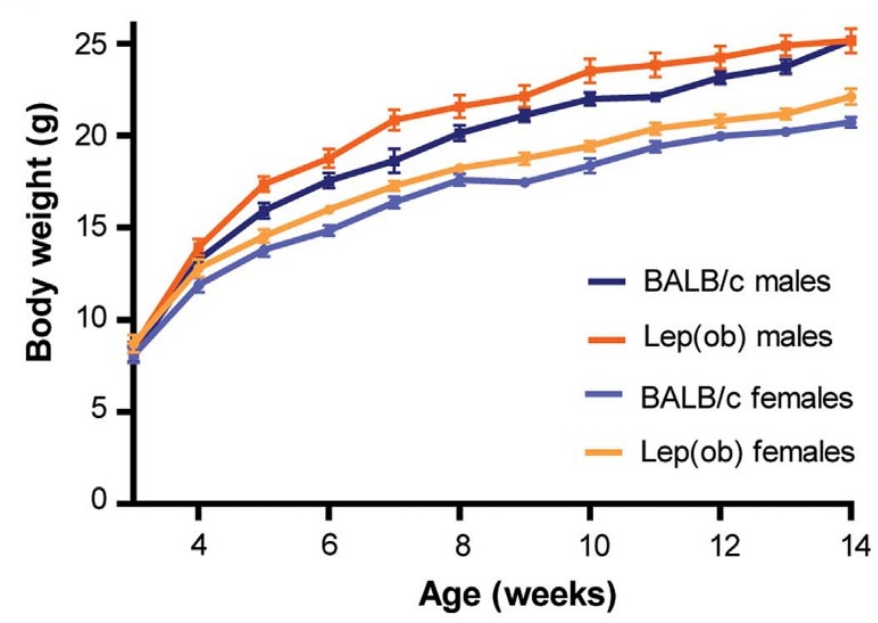

b

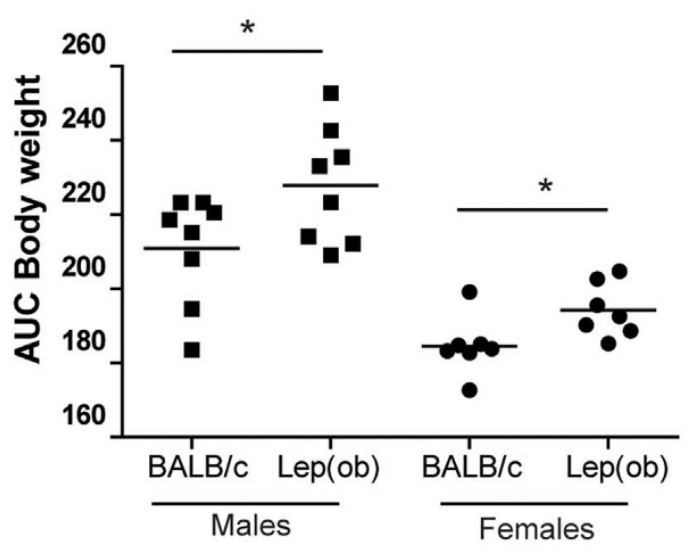

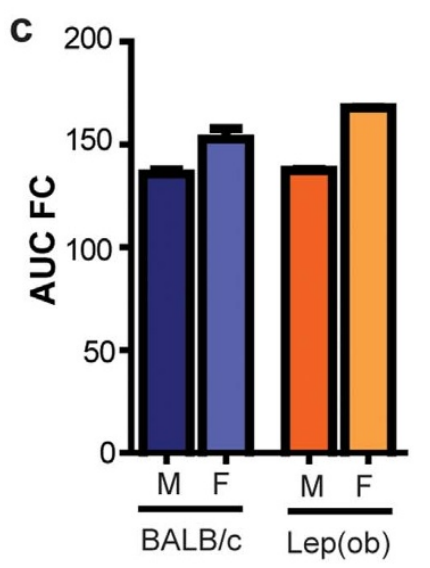
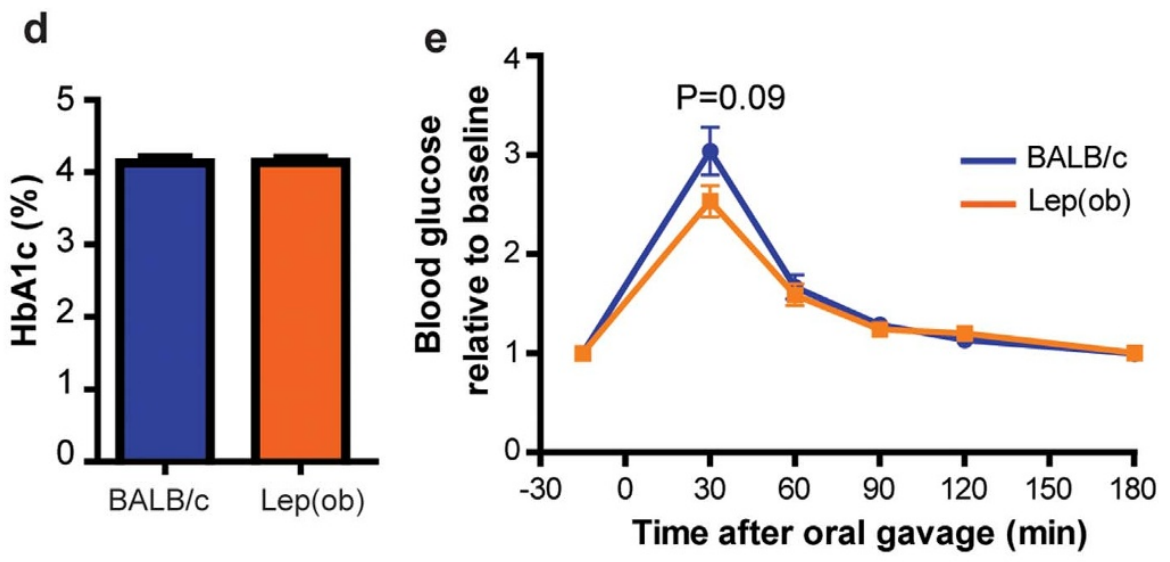

Figure 4 $\mid$ Microbiota transfer to adult antibiotic-treated mice changes their metabolic parameters. (a) Weekly body weight (gram) of recipient mice after inoculation with either BALB/c (female $n=8$, male $n=8$ ) or B6.-Lep ${ }^{o b}$ (female $n=8$, male $n=8$ ) at eight weeks of age. (b) Area under the curve calculated from weight data in A. (c) AUC of weekly food consumption (gram) per cage in six weeks from inoculation in recipient mice inoculated with either BALB/c (males $n=2$; females $n=2$ ) or B6.-Lep ${ }^{o b}$ microbiota (males $n=2$; females $n=2$ ) at eight weeks of age. (d) HbA1c (glycated haemoglobin, \%) concentration in the blood of recipient mice six weeks after inoculation with either BALB/c $(n=16)$ or B6.-Lep ${ }^{\text {ob }}(n=16)$ at eight weeks of age. (e) Oral glucose tolerance test (OGTT) six weeks after inoculation with either BALB/c $(n=16)$ or B6.-Lep $p^{o b}(n=16)$ at eight weeks of age. Tail blood glucose relative to baseline value $(-15 \mathrm{~min})$ is shown at given time points after oral gavage of glucose. Mean \pm SEM is shown. ${ }^{*}$ represents $P<0.05$; $* *$ represent $P<0.01$.

istance $^{9,16-19}$. In this study, C57BL/6 mice were inoculated with cecal contents from lean or obese mice, after depleting the majority of the existing gut microbiota by ampicillin treatment. By this we sought to investigate whether transfer of gut microbiota under conventional conditions was possible in the same manner observed in germ-free isolators, and whether or not this was dependent on age upon inoculation. Indeed, the gut microbiota in recipient mice one week after inoculation clustered according to the microbiota suspension with which they were inoculated. The similarity to the donors was however more pronounced in the mice inoculated at weaning. Furthermore, changes in gut microbiota composition between the groups persisted until six weeks after inoculation when analysed by DGGE, but the microbiota also shifted with time within the groups and, thus, the inoculum was not stable as previously documented in GF mice. It is also important to note, that the effect was only investigated in mice pre-treated with antibiotics to mimic some of the effects in GF mice. It has therefore yet to be investigated if the phenotype could be transferred to non-treated mice in the same manner.

The mice in the weaning study were likely to be under more environmental influence than in the adult study as they were raised conventionally with no attempt to sterilize cage interior, bedding, food or drinking water, enabling bacteria from the environment to colonize the gut. However, as the colonization process was equally or more successful in the weaning study, there was no advantage of autoclaving cages and assorted items. Furthermore, diversification of the mouse gut microbiota usually occurs at three weeks of age, likely due to a decrease in maternal immunoglobulin A ( IgA) supply when mice stop suckling, making this a proper time point for inoculation with gut microbiota ${ }^{20}$. Recipients in the adult study had a higher diversity of gut microbiota within the groups than the controls receiving PBS one week after inoculation, suggesting that the recipients colonize on the inoculation given. This difference was, however, diminished six weeks after inoculation, suggesting, that the mice not receiving any inoculation slowly re-colonize themselves. A significant difference in clustering was still observed between recipients of lean and obese donor microbiota six weeks after inoculation suggesting a successful long term inoculation for at least some of the introduced species. Based on the DGGE results, transfer of gut microbiota subsequent to antibiotic treatment thus seems a likely method for successful transfer of microbiota in non-germ-free conditions. GULDA analysis revealed that the major changes in the gut microbiota profile of B6-Lep ${ }^{o b}$ recipient mice compared to C57BL/6 recipient mice was a lower abundance of Bacteriodetes ${ }^{21,22}$, which was also evident in the donors. However, six weeks post inoculation recipients no longer showed these taxa specific characteristic differences 
between the lean and obese inoculated groups. It is therefore questionable how persistent the establishment of the inoculum is even though the groups still separated on the PCA plot of DGGE profiles. In a study where different strains of mice were treated with antibiotics for one week beginning at five weeks of age, their differences in microbiota were diminished, but the differences returned when antibiotic treatment was terminated and each strain got back its strain-specific microbiota within one week ${ }^{23}$, which suggest that host characteristics strongly influence the gut microbiota. However, it is important to note that GULDA analysis was only performed with the chosen bacterial primers and that the differences seen on DGGE at six weeks post inoculation could be due to other, less dominant, strains which were not analyzed by GULDA.

Even if the gut microbiota in the inoculated mice changed over time it would still be expected to influence important host functions as it has previously been shown that the gut microbiota, in an early limited window of time, can determine immune responses and glucose homeostasis later in life ${ }^{14,1,24}$. The obese phenotype has previously been shown to be transmissible with microbiota ${ }^{9}$, and similar changes was in the present study observed for the recipient mice, independent of age upon inoculation. The differences in weight and metabolic parameters were however minor compared to what was previously observed in gnotobiotic mice. It is thus possible that permanent dysbiosis is required for the obese phenotype to develop in the recipients. However, it is intriguing that despite the fact that the gut microbiota only partly resembled the donors, the phenotypes of the donors were to some degree still transmissible both at weaning and as adults. The peak in glucose concentrations in OGTT seems to be the metabolic parameter mostly affected by the change in gut microbiota in both the young and adult inoculated mice despite the minor effects on weight and fat pads. Most likely, the pancreatic $\beta$ cells of B6.-Lep $p^{o b}$ recipient mice produce more insulin upon glucose stimulation which is supported by the higher increase in insulin levels. This is interesting as hyperinsulinemia is characteristic in obesity, probably as an adaptive response of the $\beta$ cells to compensate for increased insulin resistance ${ }^{25}$. It is also possible that the gut microbiota may have a direct effect on the $\beta$ cells.

It is interesting, that co-housing mice with lean and obese microbiota has been shown to result in a transfer of gut microbiota from the lean to the obese mice which prevented weight gain, whereas the reverse transfer of microbiota from obese to lean mice was not evident $^{10}$. Also, in a human study, male subjects with metabolic syndrome were given infusions of small intestinal microbiota from lean donors, which improved their peripheral insulin sensitivity six weeks after infusion ${ }^{26}$. Consequently, it would be highly relevant to investigate the proposed conventionally raised antibiotic-treated model with lean donors and obese antibiotic-treated recipients in order to see a more pronounced transfer of the metabolic phenotype.

In conclusion, inoculation of conventional antibiotic-treated mice with gut microbiota from lean or obese donors was possible and age independent, and clustering of the inoculation groups persisted until six weeks post inoculation, but it was not stable as the compositional characteristics of the recipient microbiota changed with time. Nonetheless, although a final alternative to GF mice is not established, the minor changes in weight gain, food consumption, body fat pads, insulin production, and glucose tolerance observed are not insignificant. Further investigations using obese and non-treated recipients will further enlighten the possibility of alternatives to GF models. In addition, early life changes in gut microbiota, even in a limited time frame, have several times proven critical for development of proper immune functions and pathogenesis of chronic inflammatory diseases such as colitis and type 1 diabetes ${ }^{27,28}$. It is therefore possible, that the approach presented in this paper would have a more pronounced outcome also in other disease models.

\section{Methods}

Animals and experimental design. Experiments were carried out in accordance with the EU directive 2010/63/EU, and the Danish Animal Experimentation Act (LBK 1306 from 23/11/2007 with 2010 amendments), and approved by the Animal Experimentation Inspectorate, Ministry of Food, Agriculture and Fisheries, Denmark.

Weaning study. C57BL/6 mice (Taconic, Cat\# B6JBOM-F and B6JBOM-M) were mated and from one week before birth ampicillin (Ampivet, $1 \mathrm{~g} / \mathrm{L}$ ) was added to drinking water until the pups were weaned at three weeks of age. 9 male and 19 female pups were randomized into two groups and inoculated with cecum content from either a 12 week old B6.-Lep ${ }^{o b}$ (Taconic, kindly supplied by Umeå, Sweden) or a C57BL/6 (Taconic, Cat\# B6JBOM-M) mouse, which had been housed in the same facility for a week before they were euthanized. Each group of recipients were split into four cages. They were housed under standard conditions in open cages without filter lids and fed ad libitum with a 1324 chow diet (Altromin Cat\# 1324) and had ad libitum access to bottled water.

Adult study. C57BL/6 mice (Taconic) were born at the facility and housed in a Scantainer in autoclaved open cages with autoclaved materials. The mice were fed ad libitum with an autoclaved 1324 chow diet (Altromin, Cat\# 1324TPF) and at five weeks of age 24 female and 24 male recipient mice were treated with ampicillin (Ampivet, $1 \mathrm{~g} / \mathrm{L}$ ) in the drinking water for three weeks before they were inoculated with cecum content from either B6.-Lep ${ }^{o b}$ (Charles River, Cat\# 000632) or BALB/c mice (Taconic, Cat\# BALB-M) which had been housed in the same facility for a week before they were euthanized. One group received PBS and was thus recolonized with microbes from the environment. Each group of recipients were split into four cages (two cages containing males and two cages containing females in each group), and the water was switched back to sterilized bottled water after inoculation. All mice in both experiments were weighed weekly.

Gut microbiota transfer. Donor mice were euthanized and immediately transferred to an anaerobic chamber, in which the ceca were aseptically removed and the content was diluted 1:10 in a 50\% glycerol/PBS solution. The content was thereafter divided into aliquots and frozen in liquid nitrogen and thereafter stored at $-80^{\circ} \mathrm{C}$. At the day of inoculation the cecum solution was further diluted $1: 5$ prior to oral gavage of $0.15 \mathrm{~mL}$ per mouse.

Gut microbiota characterization. Fecal samples were collected in autoclaved eppendorf tubes and stored at $-80^{\circ} \mathrm{C}$ until further processing. Cellular DNA was extracted using the QIAamp DNA Stool Mini Kit (Qiagen, Cat\# 51504) according to manufacturer's instructions, and including a step of bead beading to optimize the DNA outcome. The DNA was amplified by means of PCR, using primers specific to the $\mathrm{V} 3$ region of the $16 \mathrm{~S}$ rRNA gene. Amplicons were randomized and thereafter analyzed by denaturing gradient gel electrophoresis (DGGE) using an acrylamide gel containing a $25 \%-65 \%$ chemical gradient (urea and formamide) that enabled separation of the PCR amplicons based on sequence differences in the V3 region. PCR and DGGE conditions are reported elsewhere ${ }^{29}$. The DGGE profiles were analyzed in Bionumerics Version 4.5 (Applied Maths) using a mix of DNA profiles as a marker.

In the weaning study, the gut microbiota was further characterized by real-time PCR based Gut Low-Density Array (GULDA), used as previously described ${ }^{30}$. Relative quantification of the following bacterial groups was included; Firmicutes, Lactobacillus spp., Clostridial clusters IV and XIV, Clostridium butyricum (cluster I), Enterococcus spp., Bacteroidetes, Bacteroides spp., B. fragilis, Parabacteroides diastonis, Prevotella spp., Alistipes spp., Bifidobacterium spp., Enterobacteriaceae, and Akkermansia muciniphila. The relative abundance of each bacterial gene target was determined by normalizing with a universal bacterial primer-target, to give a measurement of total bacteria.

Oral Glucose Tolerance Test (OGTT). The mice were fasted for six to ten hours prior to testing. At time 0 the mice were challenged with an oral dose of $50 \mathrm{mg}$ (weaning study) or $2 \mathrm{mg} / \mathrm{g}$ (adult study) glucose, and blood glucose was thereafter measured at $15,30,60,90,120$ and 180 minutes post glucose challenge. Blood was drawn by puncturing the lateral tail vein, and blood glucose was measured using a glucometer (Abbott Diabetes Care, Cat\# 70808).

Measuring glycated haemoglobin (HbA1c). A $1 \mathrm{uL}$ blood sample was taken from the lateral tail vein, and HbAlc measured by using a DCA Vantage Analyzer (Siemens Healthcare Diagnostics, Cat\# 6651932).

Body fat analysis. Fat pads (inguinal, epididymal or uterine in males and females respectively, and retroperitoneal fat pads) were collected in the weaning study and weighed on a precision electron weight.

Fasting plasma insulin measurements. At each OGTT in the weaning study blood samples for measurement of fasting plasma insulin concentration were taken 15 minutes before challenge with glucose solution, using the facial vein technique and blood was collected in EDTA coated eppendorf tubes (Bie \& Berntsen A/S, Herlev, Denmark). Plasma was stored at $-20^{\circ} \mathrm{C}$ until further analysis. Plasma insulin concentrations were measured using a Mouse Insulin ELISA kit (Mercodia, Uppsala, Sweden) according to manufacturer's protocol. 
Statistics. Statistical analysis was carried out using Minitab 16 software (Minitab Inc) and GraphPad Prism version 5.02 (GraphPad Software). In all analyses, gender, cage, and gel number were included as a factor to ensure the statistical outcome was a result of group differences and not a statistical bias. In parameters with gender difference, the statistics were made for each gender. For comparison of groups, a Students t-test or ANOVA with a Tukey or Bonferroni correction was used. For data, which were not normally distributed by the D'Agoustino test or had unequal variances a KruskalWallis or Mann-Whitney test was used. Significance of variability was evaluated by the general linear model. When repeated measurements were evaluated, repeated measurements ANOVA and post hoc Bonferroni's multiple comparisons correction were used. $P<0.05$ was considered statistically significant in all tests.

1. Mackie, R. I., Sghir, A. \& Gaskins, H. R. Developmental microbial ecology of the neonatal gastrointestinal tract. Am. J. Clin. Nutr. 69, 1035-1045 (1999).

2. Zoetendal, E. G., Akkermans, A. D. L. \& De Vos, W. M. Temperature gradient gel electrophoresis analysis of $16 \mathrm{~S}$ rRNA from human fecal samples reveals stable and host-specific communities of active bacteria. Appl. Environ. Microbiol. 64 , 3854-3859 (1998)

3. Holdeman, L. V., Good, I. J. \& Moore, W. E. C. Human fecal flora - variation in bacterial composition within individuals and possible effect of emotional stress. Appl. Environ. Microbiol. 31, 359-375 (1976).

4. Brugman, S. et al. Antibiotic treatment partially protects against type 1 diabetes in the Bio-Breeding diabetes-prone rat. Is the gut flora involved in the development of type 1 diabetes? Diabetologia 49, 2105-2108 (2006).

5. Ley, R. E., Turnbaugh, P. J., Klein, S. \& Gordon, J. I. Microbial ecology - Human gut microbes associated with obesity. Nature 444, 1022-1023 (2006).

6. Ley, R. E. et al. Obesity alters gut microbial ecology. Proc. Natl. Acad. Sci. U. S. A. 102, 11070-11075 (2005)

7. Larsen, N. et al. Gut Microbiota in Human Adults with Type 2 Diabetes Differs from Non-Diabetic Adults. Plos One 5, e9085 (2010).

8. Bäckhed, F. et al. The gut microbiota as an environmental factor that regulates fat storage. Proc. Natl. Acad. Sci. U. S. A. 101, 15718-15723 (2004).

9. Turnbaugh, P. J. et al. An obesity-associated gut microbiome with increased capacity for energy harvest. Nature 444, 1027-1031 (2006).

10. Ridaura, V. K. et al. Gut microbiota from twins discordant for obesity modulate metabolism in mice. Science 341,1241214,1-10 (2013).

11. Ding, S. et al. High-Fat Diet: bacteria interactions promote intestinal inflammation which precedes and correlates with obesity and insulin resistance in mouse. Plos One 5, e12191 (2010).

12. Macconnachie, A. A., Fox, R., Kennedy, D. R. \& Seaton, R. A. Faecal transplant for recurrent Clostridium difficile-associated diarrhoea: a UK case series. Q. J. Med. 102, 781-784 (2009)

13. Hansen, C. H. F., Metzdorff, S. B. \& Hansen, A. K. Customizing laboratory mice by modifying gut microbiota and host immunity in an early "window of opportunity". Gut Microbes 4, 241-245 (2013)

14. Hansen, C. H. F. et al. Patterns of early gut colonization shape future immune responses of the host. Plos One 7, e34043-34043 (2012).

15. Ubeda, C. et al. Vancomycin-resistant Enterococcus domination of intestinal microbiota is enabled by antibiotic treatment in mice and precedes bloodstream invasion in humans. J. Clin. Invest. 120, 4332-4341 (2010).

16. Burcelin, R., Serino, M., Chabo, C., Blasco-Baque, V. \& Amar, J. Gut microbiota and diabetes: from pathogenesis to therapeutic perspective. Diabetologica 48 , 257-273 (2011).

17. Murphy, E. F. et al. Composition and energy harvesting capacity of the gut microbiota: relationship to diet, obesity and time in mouse models. Gut 59, 1635-1642 (2010)

18. Le Chatelier, E. et al. Richness of human gut microbiome correlates with metabolic markers. Nature 500, 541-546 (2013)

19. Ellekilde, M. et al. Characterization of the gut microbiota in leptin deficient obese mice - Correlation to inflammatory and diabetic parameters. Res. Vet. Sci. 96, 241-250 (2014).
20. Inoue, R., Otsuka, M. \& Ushida, K. Development of intestinal microbiota in mice and its possible interaction with the evolution of luminal IgA in the intestine. Exp. Anim. 54, 437-445 (2005).

21. Ley, R. E. Obesity and the human microbiome. Curr Opin Gastroenterol 26, 5-11 (2010).

22. Bäckhed, F. Changes in intestinal microflora in obesity: cause or consequence? J. Ped. Gastroenterol. Nutr. 48, 56-57 (2009).

23. Vaahtovuo, J., Toivanen, P. \& Eerola, E. Bacterial composition of murine fecal microflora is indigenous and genetically guided. FEMS Microbiol. Ecol. 44, 131-136 (2003)

24. Rune, I. et al. Ampicillin-improved glucose tolerance in diet-induced obese C57BL/6NTac mice is age dependent. J. Diabet. Res. (2013).

25. Gonzalez, A. et al. Insulin Hypersecretion in Islets From Diet-Induced Hyperinsulinemic Obese Female Mice Is Associated With Several Functional Adaptations in Individual beta-Cells. Endocrinology 154, 3515-3524 (2013).

26. Vrieze, A. et al. Transfer of Intestinal Microbiota From Lean Donors Increases Insulin Sensitivity in Individuals With Metabolic Syndrome. Gastroenterology 143, 913-916 (2012).

27. Hansen, C. H. F. et al. Early life treatment with vancomycin propagates Akkermansia muciniphila and reduces diabetes incidence in the NOD mouse. Diabetologia 55, 2285-2294 (2012).

28. An, D. et al. Sphingolipids from a Symbiotic Microbe Regulate Homeostasis of Host Intestinal Natural Killer T Cells. Cell 156, 123-133 (2014).

29. Hufeldt, M. R., Nielsen, D. S., Vogensen, F. K., Midtvedt, T. \& Hansen, A. K. Family relationship of female breeders reduce the systematic inter-intervidual variation in the gut microbiota of inbred laboratory mice. Lab. Anim. 44, 283-289 (2010)

30. Bergström, A. et al. Introducing GUt Low-Density Array (GULDA) - a validated approach for qPCR-based intestinal microbial community analysis. FEMS Microbiol. Lett. 337, 38-47 (2012).

\section{Acknowledgments}

We thank Anders Bergström, DTU, for his help with the qPCR data analysis. We also thank Monika Viscardi for her help with performing the DGGE in the adult study. This work was carried out as part of the $3 \mathrm{G}$ center - Gut, Grain \& Greens. The 3G center is supported by the Danish Council for Strategic Research. It was further funded by the Center for Applied Laboratory Animal Research, CALAR.

\section{Author contributions}

M.E., E.S., F.K.V., A.K.H. and C.H.F.H. planned the experimental design. M.E., E.S., I.R. and B.T. planned and performed the animal studies and analyses performed in live animals. M.E., E.S., M.J. and D.N. performed and evaluated D.G.G.E. results. C.L., M.I.B., C.H.F.H. and T.L.R.I. performed and evaluated G.U.L.D.A. results. M.E., E.S. and C.H.F.H. wrote the manuscript. All authors reviewed and accepted the manuscript.

\section{Additional information}

Competing financial interests: The authors declare no competing financial interests.

How to cite this article: Ellekilde, M. et al. Transfer of gut microbiota from lean and obese mice to antibiotic-treated mice. Sci. Rep. 4, 5922; DOI:10.1038/srep05922 (2014).

This work is licensed under a Creative Commons Attribution-NonCommercialNoDerivs 4.0 International License. The images or other third party material in this article are included in the article's Creative Commons license, unless indicated otherwise in the credit line; if the material is not included under the Creative Commons license, users will need to obtain permission from the license holder in order to reproduce the material. To view a copy of this license, visit http:// creativecommons.org/licenses/by-nc-nd/4.0/ 